\title{
Latino/a Depression and Smoking: An Analysis Through the Lenses of Culture, Gender, and Ethnicity
}

\author{
EIma I. Lorenzo-Blanco • Lilia M. Cortina
}

Published online: 7 September 2012

(c) Society for Community Research and Action 2012

\begin{abstract}
Rates of major depressive disorder (MDD) and cigarette smoking increase with Latino/a acculturation, but this varies by gender and ethnic subgroup. We investigated how lived experiences (i.e., discrimination, family conflict, family cohesion, familismo) clustered together in the everyday lives of Latina/os. We further examined associations of cluster profile and Latino/a subgroup with MDD and smoking, and tested whether gender moderated these associations. Data came from the National Latino Asian American Study, which included 2,554 Latino/as (48\% female; mean age $=38.02$ years). K-means cluster analysis revealed six profiles of experience, which varied by gender and socio-cultural characteristics. Proportionately more women than men were in groups with problematic family lives. Acculturated Latino/as were disproportionately represented in profiles reporting frequent discrimination, family conflict, and a lack of shared family values and cohesion. Profiles characterized by high discrimination and family problems also predicted elevated risk for MDD and smoking. Findings suggest that Latino/a acculturation comes jointly with increased discrimination, increased family conflict, and reduced family cohesion and shared family values, exacerbating risk for MDD and smoking. This research on pathways to depression and smoking can inform the development of targeted assessment, prevention, and intervention strategies, tailored to the needs of Latino/as.
\end{abstract}

Keywords Acculturation - Enculturation - Gender . Smoking · Depression · Latino/as

E. I. Lorenzo-Blanco $(\varangle) \cdot$ L. M. Cortina

Departments of Psychology and Women's Studies, University

of Michigan, Ann Arbor, MI 48109-1043, USA

e-mail: elmalb@umich.edu

\section{Introduction}

Acculturation refers to the cultural, social, and psychological changes that occur in immigrant groups and individuals (Schwartz et al. 2010). The majority of US Latino/ as are immigrants or children of immigrants, making acculturation highly relevant to mental health and illness. Research indicates that markers of Latino/a acculturation are associated with higher occurrence of Major Depressive Disorder and cigarette smoking (e.g., Bethel and Schenker 2005; Vega and Sribney 2008). It is vital that we better understand why.

Approximately $15 \%$ of US Latino/as have a lifetime history of MDD (Alegria et al. 2008), and $16 \%$ report being smokers (CDC 2009). While Latina women report more depression than Latino men (Alegria et al. 2008), Latino men are more likely to smoke (CDC 2009). Moreover, Puerto Rican Americans have higher MDD and smoking prevalence compared to Mexican and Cuban Americans (Alegria et al. 2008; Pérez-Stable et al. 2001). Thus, Latino/a MDD and smoking prevalence varies by gender and ethnicity, for reasons that remain unclear. To shed light on these issues, the current project investigates how gender, ethnicity, and lived experiences that accompany acculturation jointly influence Latino/a MDD and smoking.

We focus on MDD and smoking for several reasons. MDD is one of the most burdensome diseases in the world (e.g., Andrade et al. 2003), and cigarette smoking is the leading cause of preventable death in the US (CDC 2009). Moreover, depression and cigarette smoking tend to co-occur (e.g., Pratt and Brody 2010). While some studies maintain that smokers use cigarettes as a way to selfmedicate their depressive symptoms (e.g., Breslau et al. 1998), others suggest the reverse relationship, that nicotine 
leads to depression in smokers by causing changes in their brain chemistry (e.g., Quattrocki et al. 2000). A different line of research repudiates a causal relationship between depression and smoking, proposing that depression and smoking are merely influenced by the same causal factors (Kendler et al. 1993). Regardless of the reason for their association, it seems logical that research on Latino/a MDD also addresses smoking and vice versa.

\section{Latino/a Acculturation and Enculturation}

Acculturation refers to the acquisition of cultural elements of the dominant US society. As part of this process, Latino/ as can experience changes in their attitudes, behaviors, interpersonal relationships, language, values, and ethnic identification. Specifically, Latino/a immigrants in the US adopt more individualistic values, a greater focus on interpersonal distance and independence, and an "American" identity. They also increasingly learn and speak the English language and participate in American cultural practices-such as consuming mainstream media (e.g., reading books and watching TV in the English language), having non-Latino/a white friendships, and eating American foods (Cabassa 2003; Schwartz et al. 2010).

Historically speaking, traditional models frame acculturation as a unidimensional process, in which immigrants abandon the practices, values, and identifications of their culture of origin to adopt those of the host culture (e.g., Cabassa 2003). For instance, unidimensional models assume that, as they acculturate, Latino/as lose proficiency in the Spanish language; stop consuming foods and media specific to their country of origin; reject collectivistic and Latino/a cultural values; and give up their Latino/a national or ethnic identity (Schwartz et al. 2010). Acculturation frameworks have become progressively more sophisticated over time, however.

Contemporary models of acculturation are now multidimensional, acknowledging that US Latino/as can simultaneously acculturate and enculturate. Enculturation refers to selective adherence to and acquisition of the practices, values, and identifications of Latino/a culture. With enculturation Latino/as learn or continue to use Spanish, consume foods and media from their country of origin, endorse collectivistic and Latino/a values, and continue to adhere to their Latino/a national or ethnic identity (Schwartz et al. 2010). Current thinking is that Latino/as can maintain or learn aspects of Latino/a culture (enculturation) at the same time that they acquire elements of dominant US culture (acculturation).

Culture has historically been defined as the values, norms, beliefs, and practices that pertain to a society (e.g.,
Betancourt and López 1993). Problematically, this traditional definition depicts culture as a static phenomenon residing within individuals, and portrays people as passive recipients of culture who have no agency; it overlooks the influences of the social world, daily interactions, and lived experiences in people's daily lives (e.g., Lakes et al. 2006). In contrast, contemporary research suggests that people can adhere to, modify, add to, or reject cultural elements through social processes and lived experience (López and Guarnaccia 2000). Latino/as may choose to follow some aspects of Latino/a or mainstream US culture but not others, creating diversity within Latino/a communities. In sum, research on Latino/a culture and acculturation has become increasingly nuanced over time, promising to shed new light on Latino/a depression and smoking.

\section{Latino/a Depression and Smoking}

Scholars propose that enculturation can protect Latino/as from-and acculturation can increase risk for - MDD and substance use (e.g., Grant et al. 2004). Indeed, empirical research has connected Latino/a acculturation (measured with markers of acculturation such as English proficiency, nativity, or years spent in the US) to MDD and smoking. These relationships, however, are stronger for women than men (e.g., Bethel and Schenker 2005; Vega and Sribney 2008), and they vary by Latino/a subgroup (e.g., Alegria et al. 2006, 2008). Questions remain about why some groups are more affected by acculturation than others.

Some researchers suggest that it is not acculturation or enculturation per se that lead to higher or lower risk for Latino/a MDD or cigarette smoking, but the lived experiences that accompany life in the US (Schwartz et al. 2010). In other words, lived experiences may be potential mechanisms through which markers of acculturation and enculturation link with risk for MDD and smoking. Similarly, scholars argue that it is not gender or Latino/a subgroup per se that affects risk for mental health and substance use problems, but the lived experiences associated with being female, male, Mexican, Puerto Rican, or Cuban (Cole 2009). Lived experiences such as everyday discrimination, family conflict, family cohesion, and familismo can differ for men, women, Mexicans, Puerto Ricans, and Cubans (e.g., Pérez et al. 2008; Rivera et al. 2008; Sarmiento and Cardemil 2009). The present study investigated how these experiences clustered together in the lives of Latinos and Latinas, and differed by gender, Latino/a subgroup, and other demographic characteristics. Further, we assessed how specific cluster "profiles" related to MDD and cigarette smoking. 


\section{Latino/a Lived Experiences}

\section{Everyday Discrimination}

One lived experience salient to Latino/as is everyday discrimination, defined as perceived daily experiences of unfair, differential treatment (Alegria et al. 2004). Studies suggest that Latino/a acculturation comes with more frequent encounters of everyday discrimination (Cook et al. 2009; Kam et al. 2010), and experiences of everyday discrimination vary by gender and Latino/a subgroup. Pérez et al. (2008) found higher prevalence of discriminatory encounters in Latino men than women, and Cubans had lower prevalence than Mexicans and Puerto Ricans. Moreover, discrimination relates to Latino/a MDD and cigarette smoking (e.g., Wiehe et al. 2010), possibly mediating the effects of acculturation (Cook et al. 2009; Kam et al. 2010). One theory is that, over time, discrimination influences Latino/as' mental health and substance use through stress proliferation (e.g., Alegria et al. 2004). That is, everyday experiences of discrimination can become chronic, daily stressors, which can generate additional stressful experiences. One result can be an escalation of depression and smoking (Ong et al. 2009).

\section{Family Conflict}

In addition to discrimination, Latino/as can experience more frequent family conflict when acculturating to the US (e.g., Cook et al. 2009). Family conflict among acculturating US Latino/as has been conceptualized as a form of acculturative stress, or stress that directly results from the acculturative process (e.g., Hovey and Magaña 2000). Research supports significant positive associations between acculturative stress, depression, and substance use (Hovey and Magaña 2000). Thus, family conflict may explain the associations of acculturation with higher occurrences of depression and smoking.

Scholars attribute more frequent occurrences of family conflict in part to changes in gender role endorsement, especially for Latina women, who embrace the freedom that comes with less traditional roles and therefore acculturate faster than Latino men (Gil and Vazquez 1996). Research has further shown that Latina women are more negatively affected by family conflict than their male counterparts (e.g., Sarmiento and Cardemil 2009). Moreover, experiences of family conflict vary by Latino/a subgroup, in that Puerto Ricans report the most and Cubans the least conflict (Rivera et al. 2008). There is also growing evidence of positive associations between family conflict and mental health problems among Latino/a adults (Cook et al. 2009; Sarmiento and Cardemil 2009). Family conflict could help explain why the associations of Latino/a acculturation with depression and smoking are stronger for women compared to men.

Family Cohesion and Familismo

Researchers have also documented the protective roles of Latino/a family cohesion and familismo (e.g., Rivera 2007). Family cohesion entails perceptions of family closeness and communication (e.g., Olson 1986). The cultural value of familismo emphasizes trust and family loyalty, and a general orientation to the family. It is characterized by positive family relationships, high family unity, social support, and interdependence. The strong emotional bonds measured by family cohesion and familismo are thought to promote social support from families (e.g., Rivera et al. 2008).

Consistent with the idea that social support can reduce or buffer the negative impact of stressful life events on mental health problems (e.g., Aneshensel and Frerichs 1982), family cohesion can protect Latino/as from external stress (e.g., Rivera et al. 2008). Conversely and consistent with the notion that the pure absence of social support qualifies as a stressor, research shows that low family cohesion and familismo relate to increased smoking and depressive symptoms in Latino/as (Rivera 2007; Rivera et al. 2008; Coonrod et al. 1999). Also, as acculturation increases, family cohesion and familismo decrease among Latino/as (e.g., Miranda et al. 2000; Baer and Schmitz 2007). Moreover, Latino/a family cohesion varies by ethnic subgroup, with Cubans reporting the highest levels and Puerto Ricans reporting the lowest (Rivera et al. 2008). Less is known about gender differences in experiences of family cohesion and familismo.

\section{Towards a Holistic Understanding of Lived Experiences}

In all, researchers have demonstrated the significant roles played by discrimination, family conflict, family cohesion, and familismo in the mental health and substance use of Latino/as from diverse backgrounds. Although this understanding is important, it is also fragmented, with each study examining the influence of only one or possibly two lived experiences. In real life, instances of discrimination, family conflict, and family cohesion co-occur, jointly influencing Latino/a well-being. An important next step is for research to take a holistic view of these lived experiences, and investigate how different combinations or "profiles" of experience influence MDD and cigarette smoking.

In other words, past studies have relied on variablecentered research methods by treating each kind of lived 
experience as an isolated entity, thereby reducing complex and dynamic phenomena into smaller elements (Magnusson 1998). In person-centered approaches, in contrast, the unit of analysis is the individual's lived experiences as an organized whole (Magnusson 1998). As such, person-centered methods can provide a more holistic and multifaceted view of how experiences come together to create diverse pathways to MDD and cigarette smoking in US Latino/as. This understanding is important because US Latino/as are not only the largest and fastest-growing immigrant group in the US (US Census Bureau 2010), they are also a diverse group of people with different life experiences, socio-political histories, and socio-cultural backgrounds (Aguilar-Gaxiola et al. 2008). Further, research on pathways to Latino/a MDD and smoking can inform the development of targeted prevention, intervention, and policy-making strategies.

In this study, we investigated how specific lived experiences (i.e., everyday discrimination, family cultural conflict, family cohesion, and familismo) clustered together in the everyday lives of Latinas and Latinos. We also compared cluster "profile" groups on demographic and socio-cultural variables including gender, ethnicity, language, and years spent in the US, among others. Moreover, we assessed how lived experience profiles related to MDD and cigarette smoking. All analyses considered the role of gender and ethnicity, because studies suggest that lived experiences can differ for men, women, and individuals from different Latino/a subgroups. Based on prior (variable-centered) research, we hypothesized that profiles distinguished by frequent family conflict would contain disproportionately more women than men. Moreover, we predicted that more men than women would belong to profile groups describing frequent discrimination. Further, we expected disproportionately more Cubans to be in profile groups reporting low discrimination, low family conflict, and high family cohesion. In addition, we hypothesized that profile group and Latino/a subgroup would relate significantly to MDD and smoking, with gender moderating these associations. Generally, we expected groups characterized by high discrimination and family conflict as well as low family cohesion and familismo to be most at risk. These analyses controlled for the influence of education and income, to rule these out as alternative explanations for elevated risk.

\section{Methods}

Sample and Procedure

Data came from the National Latino and Asian American Study (NLAAS), a nationally representative household survey of non-institutionalized Latino/a and Asian adults, residing in the conterminous US. The NLAAS was designed to gather psychiatric information comparable to psychiatric information collected by the National Comorbidity Survey Replication (NCS-R) and the National Study of American Life (NSAL) (e.g., diagnostic measures of depression and anxiety). It also assessed a range of environmental and socio-cultural factors and experiences unique to Asian Americans and Latino/a Americans in the US (Alegria et al. 2004). Respondents completed NLAAS interviews in the language of their preference. The final sample included 2,554 Latino/as (weighted response rate of $75.5 \%$ ), in addition to 2,095 Asian Americans. For further sampling details, see Heeringa et al. (2004).

We limited our analysis to the Latino/a subsample: 868 Mexicans, 577 Cubans, 495 Puerto Ricans, and 614 "Other Hispanics." Approximately $57 \%$ of these Latino/as was born outside the US, $48 \%$ were female, and the mean age was 38 years. Thirty-nine percent of the subsample had completed 11 years of education or less, and $12 \%$ had completed at least 16 years of education. Over $60 \%$ were employed, and $64 \%$ were married.

\section{Measures}

\section{Lifetime and Past-Year MDD}

Lifetime and past-year history of Major Depressive Disorder (MDD) were assessed with the diagnostic interview of the World Mental Health Survey Initiative version of the World Health Organization Composite International Diagnostic Interview (WMH-CIDI; Kessler and Ustun 2004), a structured diagnostic instrument based on DSMIV criteria. Based on this interview, participants received scores of either 1 (meets criteria) or 0 (does not meet criteria) on Lifetime $M D D$, and either 1 or 0 on Past-Year $M D D$.

\section{Lifetime and Current Smoker Status}

Smoker status was established by asking individuals whether they were current smokers, ex-smokers, or never smokers. We dichotomized response options in two ways, to indicate Lifetime Smoking $(0=$ Never Smoker, $1=$ Lifetime Smoker) as well as Current Smoking $(0=$ Not a Current Smoker, $1=$ Current Smoker $)$.

\section{Everyday Discrimination}

Everyday discrimination was measured with nine items adopted from the Detroit Area Study (Alegria et al. 2004). Sample items included: (1) You are treated with less respect than other people, (2) People act as if they think you are not smart, and (3) You receive poorer service than 
other people at restaurants or stores. Respondents indicated the frequency of each experience on a 6-point scale, ranging from $1=$ never to $6=$ almost every day. Higher scores represented higher discrimination (Cronbach's $\alpha=.91)$.

\section{Family Conflict}

Family conflict was measured with a 5-item scale taken from the family/culture stress subscale of the Hispanic Stress Inventory (Alegria et al. 2004). Sample items include: (1) Because of the lack of family unity, you have felt lonely and isolated, (2) Your personal goals have been in conflict with your family, and (3) Because you have different customs, you have had arguments with other members of your family. Respondents reported the frequency of each experience on a 3-point scale $(1=$ hardly ever or never, $2=$ sometimes, and $3=$ often). Higher scores represented higher levels of family conflict (Cronbach's $\alpha=.79$ ).

\section{Familismo}

Seven items taken from the Family Environment Scale (Olson 1986) gauged familismo. Sample items include (1) Family members respect one another, (2) We share similar values as a family, and (3) We can express our feelings with our family. Respondents indicated how strongly they agreed or disagreed with each statement on a scale from 1 (strongly disagree) to 4 (strongly agree); higher scores indicated higher levels of familismo (Cronbach's $\alpha=.91$ ).

\section{Family Cohesion}

Three items assessed family cohesion: (1) Family members like to spend free time with each other, (2) Family members feel very close to each other, (3) Family togetherness is very important (Olson 1986). Respondents indicated their agreement with each statement on a 4-point scale, ranging from $1=$ strongly disagree to $4=$ strongly agree, with higher scores indicating higher levels of family cohesion (Cronbach's $\alpha=.83$ ).

\section{Gender}

Gender was self-reported and dummy coded as $1=$ female and $0=$ male.

\section{Ethnicity}

Respondents self-identified their ethnic background as one of the following: Cuban, Puerto Rican, Mexican or Other Hispanic.

\section{Nativity}

In a single item, respondents indicated their nativity as either born in the US (which we coded as 0) or born in another country (coded as 1).

Spanish and English Proficiency

Spanish proficiency was measured with three items from the Cultural Identity Scales for Latino/a Adolescents (Felix-Ortiz et al. 1994). Respondents indicated how well they speak, read, and write in the Spanish language (from $1=$ poor to $4=$ excellent). Scores were summed, and higher scores represented better Spanish proficiency (Cronbach's $\alpha=.90$ ). A parallel measured was created specifically for the NLAAS to assess English proficiency (Cronbach's $\alpha=.97$ ).

\section{Years Spent in the US}

We coded respondents' years spent in the US on a 5-point scale: $1=$ less than 5 years, $2=$ five to ten years, $3=11$ 20 years, $4=20$ years or more, and $5=U S$ born. Thus, higher scores represented more years spent in the US.

\section{Age of Immigration}

Foreign-born participants reported their age of US immigration, which we coded on a 5-point scale: $1=35$ years or older, $2=18-34$ years, $3=13-17$ years, $4=$ less than 12 years, and $5=$ US born. Higher scores represented younger age at immigration.

\section{Other Demographics}

Respondent's marital status was coded as married/cohabiting $=1$, divorced/separated/widowed $=2$, and never married $=3$. Employment was coded as $1=$ employed, $2=$ unemployed, and $3=$ not in the labor force. Education was measured with the following ordered categories, coded such that higher scores indicate more education: 0-11 years of education, 12 years, 13-15 years, and 16 or more years. Respondents indicated their age in years. Income was measured as "household income," and ranged from $\$ 0$ to $\$ 200,000$.

\section{Results}

\section{Descriptive Findings}

Table 1 shows weighted summary statistics for dependent and independent variables for the full sample $(N=2,554)$, 


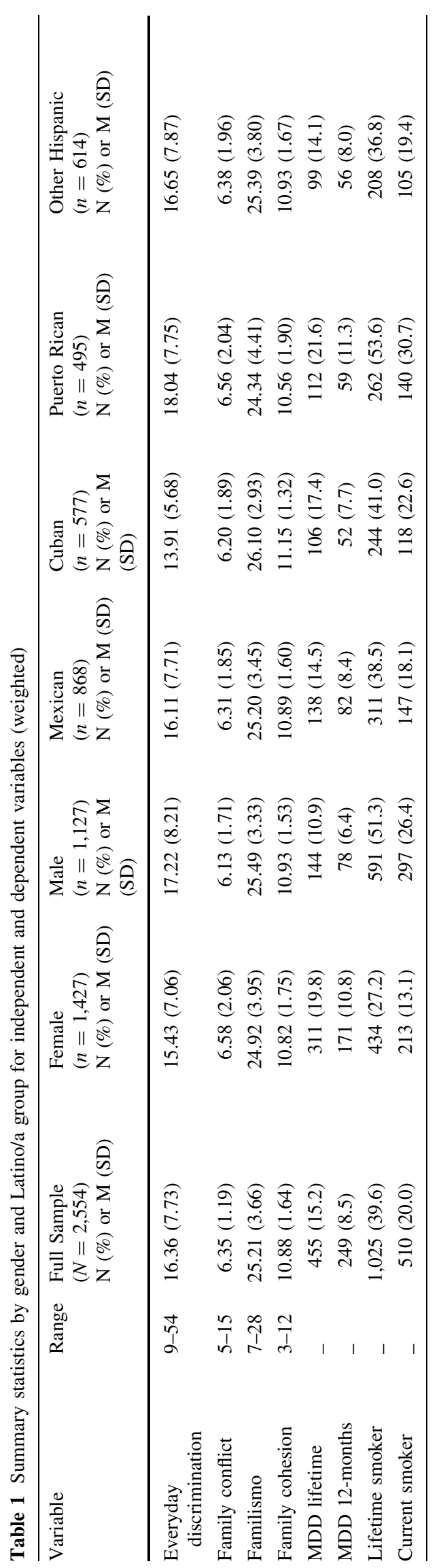

by gender (male, female), and by Latina/o subgroup (Mexican, Cuban, Puerto Rican, and Other Latino/a). As shown, approximately $15 \%$ of the full sample endorsed a history of lifetime MDD, and almost $9 \%$ met criteria for past-year MDD. The mean age of MDD onset was 25.35. Women were almost twice as likely to meet criteria for lifetime MDD (19.8\%) compared to men (10.9\%), and Puerto Ricans had higher lifetime prevalence of MDD $(21.6 \%)$ compared to Cubans $(17.4 \%)$, Mexicans $(14.5 \%)$, and the "Other Hispanic" group (14.1\%). We observed a similar pattern for past-year MDD prevalence (women higher than men, and Puerto Ricans higher than other Latino/as).

Nearly $40 \%$ of the full sample endorsed lifetime smoking, with a mean age of smoking onset being 15.21 years. The lifetime smoking prevalence for men (51.3\%) was almost twice the prevalence for women (27.2\%), and Puerto Ricans (53.6\%) had the highest lifetime smoking prevalence followed by Cubans (41.0\%), Mexicans (38.5\%), and the other Hispanic group (36.8\%). Moreover, $20.0 \%$ of Latino/as were current smokers, with more current smoking among men compared to women, and Puerto Ricans compared to other ethnic groups.

\section{Profiles of Lived Experiences: Cluster Analysis}

Next, we used cluster analysis to classify individuals into profile groups based on their lived experiences (i.e., everyday discrimination, family conflict, familismo, and family cohesion). For the entire sample, we began by standardizing our four lived experience variables, and then submitting these standardized data to $k$-means analysis (Hartigan 1975). This technique partitions cases into $n=k$ clusters by maximizing between-cluster differences and minimizing withincluster variance. According to Hartigan (1975), the number of clusters (i.e., $k$ ) should not be decided in advance, and the $k$-means algorithm should be run with several different values of $k$, chosen at random. We requested two- through seven-cluster solutions, retaining the six-cluster solution for further analysis. The six-cluster solution captured the widest variety of profiles while maintaining sufficiently large cell sizes for meaningful analyses.

Figure 1 shows the means on the z-scored lived experiences and (in the legend) the sample size for each of the six profile groups. According to this figure, members of Group $1(n=1,224)$ reported the least discrimination and family conflict, and the highest levels of familismo and family cohesion. In other words, Group 1 was distinguished by having the lowest stress and most positive family lives. Group $2(n=333)$ individuals described low discrimination, low family conflict, low familismo, and low family cohesion. That is, Group 2 members reportedly lacked both stress and positive family factors (i.e., low stress, low 


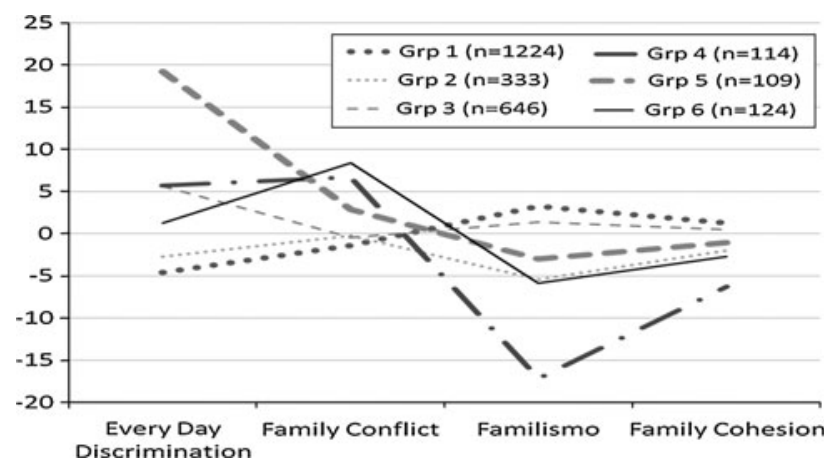

Fig. 1 Profile of mean lived experiences for each profile group. Group 1 = Lowest stress, highest positive factors, Group $2=$ Low stress, low positive factors, Group $3=$ High discrimination, low conflict, some positive factors, Group $4=$ High discrimination, high family conflict, lowest positive factors, Group $5=$ Very high discrimination, average conflict, average positive factors, Group $6=$ Highest conflict, some discrimination, average positive factors

positive factors). Individuals in Group $3(n=646)$ detailed high levels of discrimination, low family conflict, and high familismo and family cohesion. Thus, Group 3 members were characterized by high discrimination in the presence of some positive family factors (i.e., high discrimination, low conflict, some positive factors). Group $4(n=114)$ individuals were characterized by high discrimination, high family conflict, very low familismo, and very low family cohesion (i.e., high discrimination, high family conflict, lowest positive factors). Group $5(n=109)$, the smallest group, contained individuals with very high levels of discrimination, but average levels of family conflict, familismo, and family cohesion (i.e., very high discrimination, average conflict, average positive factors). Group 6 $(n=124)$ was characterized by the highest levels family conflict, average familismo and family cohesion, and some discrimination.

For the remainder of the analyses, we used Group 1 as the reference category, because Group 1 scored the lowest on discrimination and family conflict while scoring the highest on protective factors. As such, Group 1 seemed likely to have the lowest risk for MDD or smoking.

\section{Demographics of Profile Groups: Weighted Chi-Square and Wald-F Tests}

To determine whether profile groups differed as a function of demographic and socio-cultural characteristics, we conducted weighted Chi-square and Wald-F tests (testing associations with categorical and continuous variables, respectively). With weighted Chi-square tests, we found significant differences between profile group membership and gender, $\chi^{2}(d f=3.81, n=2,550)=51.74, p<.001$; Latina/o group, $\quad \chi^{2} \quad(d f=7.42, \quad n=2,550)=49.17$, $p<.05$; nativity, $\chi^{2}(d f=3.89, n=2,549)=126.15$, $p<.001$; and marital status, $\chi^{2}(d f=4.90, n=2,550)=$ 93.29, $p<.005$. With weighted Wald-F tests, we found significant differences between profile groups on English proficiency, $F(5,49)=15.78 p<.001$; Spanish proficiency, $F(5,44)=19.90, p<.001$; years spent in the US, $F(5,49)=4.22, \quad p<.05 ; \quad$ education, $F(5,49)=10.36$, $p<.001 ;$ age $F(5,49)=18.93, p<.001 ;$ and income, $F(5,49)=7.43, p<.001$.

Table 2 presents weighted demographic statistics for each of the six profile groups and the full sample. A review of profiles allowed us to consider gender differences within and between profile groups, assessing differences in lived experiences for men and women. Large gender differences emerged in Groups 3, 4, 5, and 6. Groups 4 and 6 (which reported the lowest familismo and family cohesion of any groups) were made up of nearly $60 \%$ women. Group 3 (high discrimination, low family conflict, some familismo, some family cohesion) contained about $60 \%$ men, and Group 5 (very high discrimination, average conflict, average positive factors) consisted of almost $70 \%$ men.

The ethnic make-up of each profile group largely reflected that of the full sample. However, Puerto Ricans were disproportionately more likely to appear in Group 4 (high discrimination, high family conflict, lowest positive factors) than in any of the other groups.

In regard to nativity, we found that Group 1 (the group with the least discrimination and most positive family lives) contained proportionately more foreign-born Latina/os (68\%) than any other group. Group 4 (which reported high discrimination and the least positive family lives) contained disproportionately more US born Latino/as (67 \%) than any other group. Similarly, a disproportionately high percentage of US born Latina/os (64 \%) emerged in Group 5 (the group describing by far the most discrimination).

Regarding indicators of acculturation and enculturation, individuals in Groups 3, 4, and 5 (which had faced the most discrimination, but varied on other factors) reported higher English- language proficiency than individuals in the other groups. In contrast, Group 1 (least stress, most positive factors) and Group 6 (highest family conflict, some discrimination and positive factors) reported higher Spanish proficiency than the other groups. We also observed that individuals in Groups 3, 4, and 5 had spent the longest amount of time in the US, while those in Groups 1 and 6 had spent the least time in the US Moreover, participants who had immigrated to the US in childhood (i.e., age 12 or earlier) were disproportionately overrepresented in Groups 3, 4, and 6, and underrepresented in Group 1. The opposite pattern emerged for individuals who had immigrated in early adulthood, between the ages of 18-34 (i.e., overrepresented in Group 1, and underrepresented in Groups 3, 4, 5, and 6).

Differences in marital status by profile group were particularly apparent for never-married individuals, who 


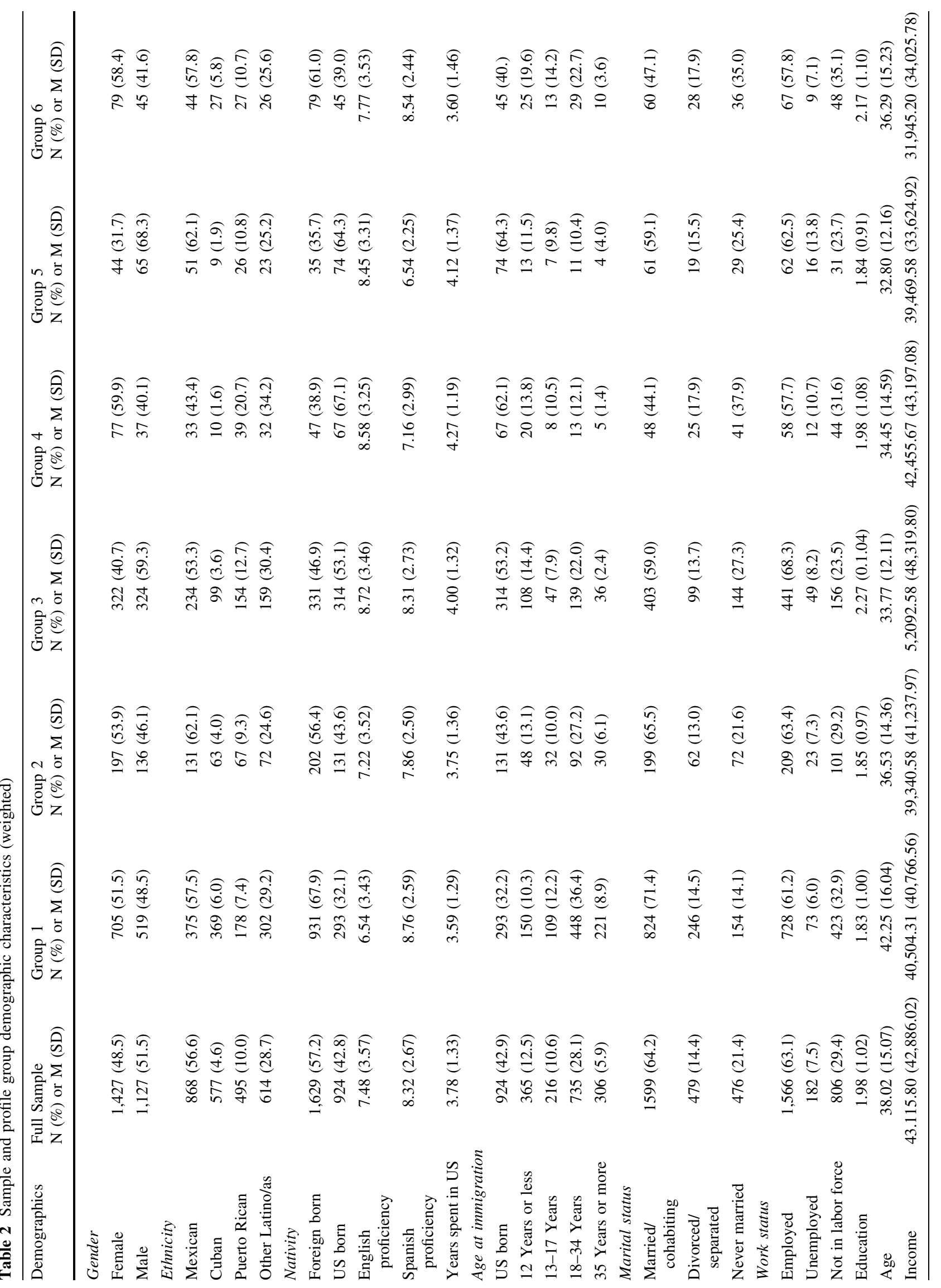




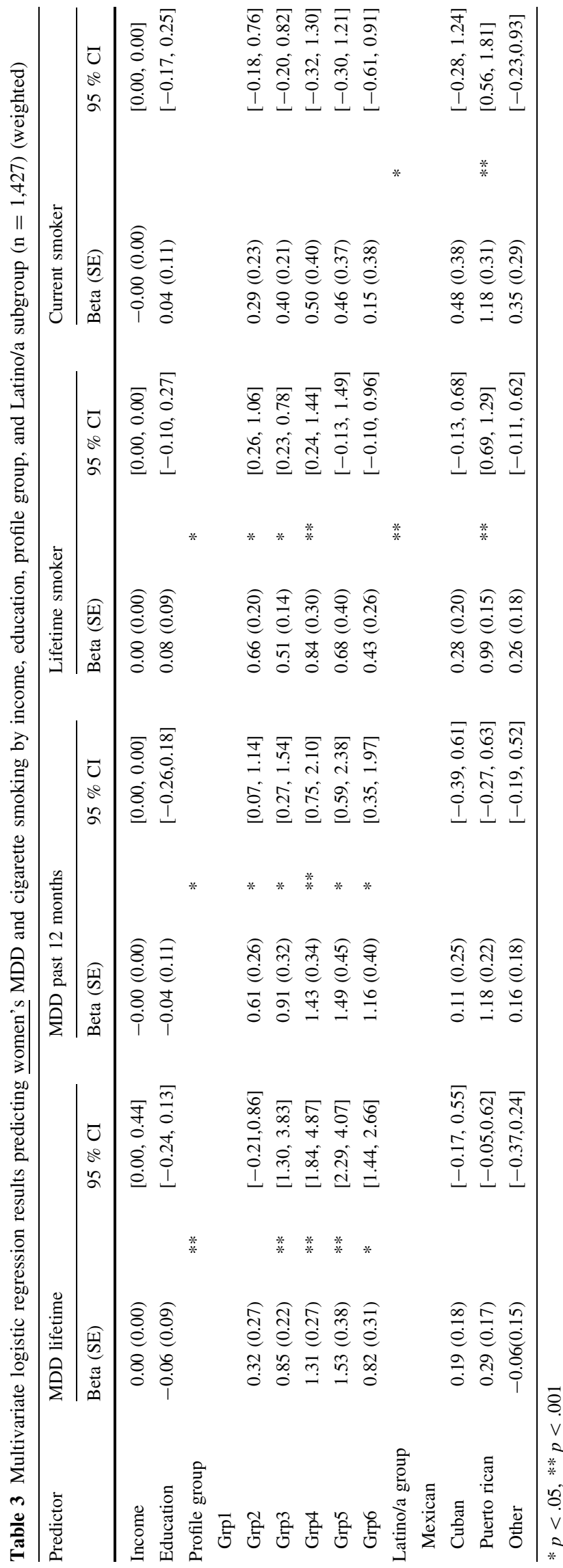

were disproportionately underrepresented in Group 1, and overrepresented in Groups 3, 4, and 6. Regarding differences in work status by profile group, it stood out that unemployed individuals were disproportionately overrepresented in Group 5. In terms of age and group membership, members of Group 1 were older than other individuals, with an average age of 42. Average income was highest in Group 3, and lowest in Group 6.

In sum, we identified six profile groups, each characterized by a unique combination of lived experiences (i.e., discrimination, family conflict, familismo, and family cohesion). We observed that profile groups differed as a function of demographic and socio-cultural experiences, including gender, Latino/a subgroup, nativity, language proficiency, years spent in the US, age at immigration, education, income, and marital status. Of note, Latina/os facing the most discrimination (Group 5) were disproportionately male, US born, proficient in English, and unemployed. They were also among those who had spent the most years in the US and immigrated at younger ages. Conversely, Latina/os experiencing the least discrimination and most positive family lives (Group 1) were disproportionately foreign-born, proficient in Spanish, married or partnered, and older; this group had spent the least amount of time in the US, and was most likely to have immigrated in young adulthood.

Predictors of MDD and Cigarette Smoking: Weighted Multivariate Logistic Regression Analyses

Lastly, we used weighted multivariate logistic regression to examine the associations of profile group and Latina/o ethnicity with our outcome variables (MDD and smoking), controlling for income and education. We stratified these regressions by gender, to determine whether gender moderated any relationships. Unfortunately, limitations in sample size did not allow us to include interaction terms in our weighted logistic regressions. Table 3 shows the results of regression analyses for women, and Table 4 shows results for men.

\section{Lifetime and Past-Year MDD}

As shown in Table 3 (columns 1 and 2), only profile group was significantly associated with lifetime and past-year MDD among women. Specifically, women in groups characterized by high discrimination and/or family conflict (i.e., Groups 3, 4, 5, and 6) were more likely to have had a lifetime history of MDD compared to women in Group 1 (the group with the least stress and highest positive factors). Women in every group were also more likely to meet pastyear MDD criteria compared to women in Group 1. These findings suggest that Latina women's risk for developing 


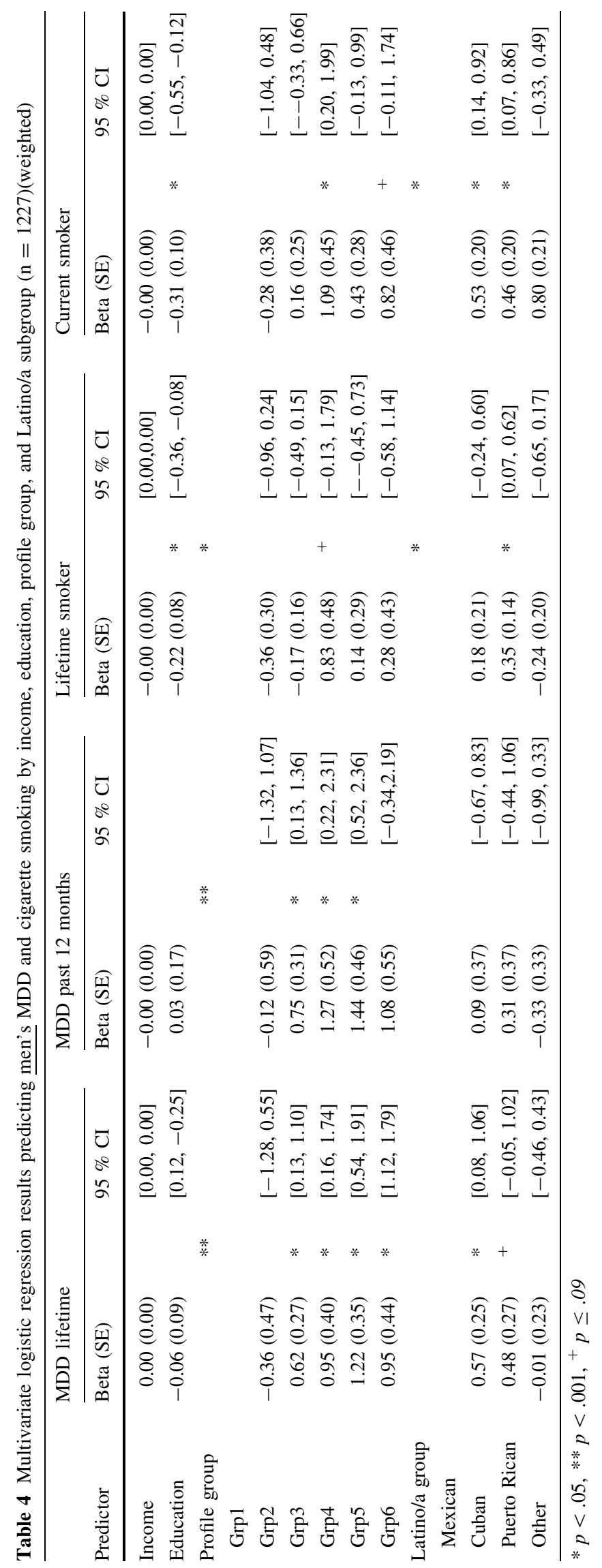


depression depends heavily on their experiences with everyday discrimination and with their families. In contrast, Latina women's MDD appears relatively unaffected by their income, education level, and ethnic subgroup.

For men (Table 4, column 1), profile group and Latino/a subgroup were both significantly associated with lifetime MDD. Similar to the results for women, men in groups experiencing moderate- to high-frequency discrimination (i.e., Groups 3, 4, 5, and 6) were at elevated risk for lifetime MDD compared to men in Group 1, who had encountered the least discrimination and most positive family lives. Moreover, Cuban American and Puerto Rican men (unlike women) were more likely to have had a lifetime history of MDD compared to Mexican-American men, although the effect for Puerto Ricans was only marginally significant $(p=.08)$. Profile group was the only significant predictor of past-year MDD for men (Table 4, column 2). Men in Groups 3, 4, and 5 (the most discriminated-against groups) were more likely to meet past-year MDD criteria compared to men in Group 1. In sum, life experiences characterized by frequent discrimination were associated with increased risk for depression (both recent and lifetime) in men. Discrimination therefore appears to be especially detrimental to Latino/a men's mental health. Income and education levels, however, had no effect.

\section{Lifetime and Current Smoker Status}

Profile group and Latino/a subgroup were significantly associated with lifetime smoking in women (Table 3, column 3). Women in Groups 2 (i.e., low stress, low positive factors), 3 (i.e., high discrimination, average conflict, some positive factors), and 4 (i.e., high discrimination, high family conflict, lowest positive factors) were more likely to have smoked cigarettes at some point in their lives compared to women in Group 1 (i.e., lowest stress, highest high positive factors). Puerto Rican women also reported more lifetime smoking than Mexican-American women. In regard to women's current smoking (Table 3 column 4), only Latino/a subgroup was significantly associated with current smoker status, with Puerto Rican women being more likely to smoke, compared to Mexican women. Income and education levels showed no relationship to women's smoking.

Profile group and Latino/a subgroup were also significantly associated with lifetime smoking in men (Table 4, column 3). Men in Group 4 (i.e., high discrimination, high family conflict, lowest positive factors) were more likely to be lifetime smokers compared to men in Group 1, but the effect was only marginally significant $(p=0.09)$. Paralleling the results for women, Puerto Rican men were more likely to be lifetime smokers compared to Mexican men. In contrast with the women's results, however, men's education was associated (negatively) with lifetime smoking. For men, profile group, Latino/a subgroup, and education were also significantly associated with current smoker status (Table 4, column 4). Group 4 men were significantly more likely to smoke currently than Group 1 men. Moreover, Cuban and Puerto Rican men were more likely to smoke currently than Mexican men, and education again related negatively to current smoker status. In sum, Latino men's reported smoking increased with lower education, Puerto Rican ethnicity, and experiences of frequent discrimination and family conflict in the absence of shared family values, closeness, and cohesion.

Comparing findings across Tables 3 and 4 , it is interesting that profile Group 4 (the group with the lowest familismo and family cohesion) was associated with lifetime smoking in women and current smoking in men; this elevated smoking risk did not emerge, however, for Group 6 (which differed from Group 4 only in having more positive family lives). Group 5 women and men (who also described more positive family lives than Group 4, but also mисh more discrimination) showed no increased smoking at all. Moreover, membership in Group 2 (which had experienced low stress, but also low familismo and family cohesion) predicted lifetime smoking in women, but not men. These patterns suggest that the absence of shared family values and family cohesion correlates with elevated smoking risk among Latino/as, especially women.

\section{Discussion}

Based on a large national sample of US Latino/as, this study took a person-centered approach to understand how gender, culture, discrimination, and family converge in everyday Latino/a lives, creating unique pathways to MDD and cigarette smoking. Latino/as are exposed to a multitude of acculturated-related experiences simultaneously, which can either increase or decrease risk for depression and substance use. We build on previous work to document how those lived experiences combine and covary, yielding different life profiles. Some profiles related to depression and smoking while others did not, and many relationships differed by gender. We now discuss key findings.

\section{Profiles of Lived Experience}

$\mathrm{K}$-means cluster analysis illustrated the diverse nature of lived experiences among Latino/as in the US. We found six distinct profiles of experiences, which ranged from low discrimination and highly positive family lives, to high discrimination and frequent family conflict, to low discrimination, low conflict, and low shared family values. These distinct profiles showed systematically that not all 
Latino/as experience stress, and not all Latino/as have access to the same protective cultural practices and values. Overall, k-means analysis proved a useful tool for demonstrating Latino/a diversity.

After identifying the different profiles, we reviewed their demographic composition. The profile groups differed by gender, language proficiency, nativity, years spent in the US, and age at immigration. Proportionately more women than men were found in groups characterized by problematic family lives (i.e., high family conflict, low family cohesion, low familismo), which supports the notion that family tension may be more relevant for Latina/o women than men. Scholars have proposed that family conflict is a result of changes in traditional gender roles during the acculturation process (Gil and Vazquez 1996). Moreover, researchers have hypothesized that immigrant women acculturate faster than immigrant men, creating a mismatch in gender-role expectations between men and women. This ultimately leads to family cultural conflict (Gil and Vazquez 1996), and women may feel guilty for putting family harmony at risk.

We further found acculturated women, compared to less acculturated women, not only reported elevated family conflict but also discrimination. Profile Groups 4 (high discrimination, high family conflict, lowest positive factors) and 6 (highest conflict, some discrimination, average positive factors) (i.e., the two groups with proportionately more women than men) were characterized by similar amounts of family conflict. However, individuals in Groups 4 and 6 differed in regard to acculturation and discrimination. Group 4 (high discrimination, high family conflict, lowest positive factors) appeared to be more acculturated than Group 6 (highest conflict, some discrimination, average positive factors); that is, Group 4 individuals were more English proficient, had spent more time in the US, and were more likely to be US born. Moreover, compared to Group 6, Group 4 was characterized by high discrimination, in addition to high family conflict. These results indicate that it is not only family conflict that can accompany Latina women's acculturation, but also discrimination.

More generally, acculturated Latino/as (i.e., individuals in Groups 3, 4, and 5, who were more English proficient, had spent more time in the US, and/or were more likely to be US born) reported more discrimination than lessacculturated Latino/as (i.e., individuals in Groups 1, 2, and 6). This suggests that acculturation may expose Latino/as to discriminatory practices, and it supports findings from prior research. Researchers have proposed different rationales for the association between Latino/as acculturation and discrimination. One perspective suggests that more acculturated Latino/as (i.e., Latino/as who were born in the US, have spent more time in the US, and/or speak more English) encounter more discrimination because they have more opportunities for exposure (Agnew 2001). Others argue that acculturated Latino/as are more aware of ethnic disparities and hierarchies present in the US, and as a result they perceive greater discrimination (Guilamo-Ramos et al. 2004). Both perspectives could be valid.

\section{Depression and Smoking}

In total, $15 \%$ of the sample reported a history of lifetime MDD, and almost $9 \%$ met criteria for past-year MDD. Approximately $40 \%$ were lifetime smokers, and around $20 \%$ were current smokers. As in prior studies, gender differences emerged, with more women experiencing depression and more men smoking. To better understand life circumstances surrounding Latino/a risk for depression and smoking, we examined how these outcomes varied by profile group and ethnic subgroup. Stratifying this analysis by gender, we found both differences and similarities between women and men.

Two profile groups (4 and 6) stood out as having the most difficult family experiences, (i.e., the most family conflict, least family cohesion, and lowest familismo), and these profiles were associated with elevated risk. Specifically, both women and men in Group 4 (high discrimination, high family conflict, lowest positive factors) showed increased vulnerability to depression and smoking, and Group 6 (highest conflict, some discrimination, average positive factors) was associated with depression in both genders (past-year and lifetime MDD in women, and lifetime MDD in men). In all, these findings suggest that improving Latino/a family functioning could help protect against depression and smoking, in both men and women. Scholars have theorized that family conflict adversely affects Latina females' mental health and substance use (e.g., Sarmiento and Cardemil 2009), and the current study extends that conclusion to Latino men.

Reducing discrimination can also benefit Latino/a mental health. The three groups characterized by high discrimination (i.e., Groups 3, 4, and 5) were significantly more likely to have had a history of MDD (lifetime and past-year) compared to Group 1 (lowest stress, highest positive factors). Further, Groups 3 (high discrimination, low conflict, some positive factors) and 4 (high discrimination, high family conflict, lowest positive factors) were associated with lifetime smoking in women, and Group 4 (high discrimination, high family conflict, lowest positive factors) was associated with current smoking in men. This study assessed the "everyday" variety of discrimination (e.g., being treated with less respect than others, receiving poorer service). These experiences may appear trivial at first glance, especially when compared to more blatant forms of discrimination (e.g., in employment, college admissions). Our findings, however, suggest that even subtle discrimination can have adverse mental health consequences for Latino/as, both male and female. 
Group 1 (lowest stress, highest positive factors) individuals described the least amount of stress and most positive family lives. Moreover, when comparing Latino/as in Group 1 (lowest stress, highest positive factors) to those in Group 6 (highest conflict, some discrimination, average positive factors), we observed that Group 1 (lowest stress, highest positive factors) was more enculturated and less acculturated. That is, Group 1 individuals were more Spanish-proficient and less English-proficient, had spent less time in the US, and were more likely to be foreign born than US born. Further, compared to Group 1(lowest stress, highest positive factors), Group 6 (highest conflict, some discrimination, average positive factors), reported more family conflict, more discrimination, less familismo, and less family cohesion. The differences in lived experiences between Groups 1 (lowest stress, highest positive factors) and 6 (highest conflict, some discrimination, average positive factors) may indicate that as Latino/as acculturate to the US, they lose protective factors such as shared family values and family closeness, while at the same time they experience more stress in the form of family conflict and everyday discrimination. Also, compared to Group 1 (lowest stress, highest positive factors), Group 6 (highest conflict, some discrimination, average positive factors) was more likely to have a history of MDD. These findings suggest that acculturation increases Latino/as' depression risk, perhaps due to increased discrimination and family conflict as well as loss of cultural values and family cohesion. The combination of high discrimination, high family conflict, lack of familismo, and lack of family cohesion seems to be particularly detrimental for women (profile Group 6 was associated with lifetime and past-year MDD for women, but only with lifetime MDD in men). This points to the need for interventions to prevent depression in Latinas with this risk profile.

Profile Group 2 (low stress, low positive factors) was made up of individuals with similar proficiency in both English and Spanish, and there were no large nativity differences. It is possible that this group largely consisted of people who were bi-cultural. Scholars (e.g., Schwartz et al. 2010) argue that bi-cultural individuals are able to effectively navigate aspects of the US and Latino/a culture, and as a result, they encounter less discrimination and family conflict than those who are mono-cultural. Research has also shown that bi-cultural individuals have better mental and physical health than individuals who more strongly identify with one culture (Schwartz et al. 2010). The results of our study support this notion, but only among men. In contrast, Group 2 women were more likely to smoke and have had a history of MDD than Group 1(lowest stress, highest positive factors) women (who appeared less acculturated and thus more mono-cultural). These findings raise interesting questions about whether and why biculturalism benefits Latinos but not Latinas. They also illustrate the need to investigate Latino/a mental health through a lens of gender.

Interestingly, Latino/a subgroup was associated with smoking and MDD, and these associations varied by gender. Puerto Rican women and men were more likely to endorse a history of smoking compared to Mexican women and men. Moreover, Cuban and Puerto Rican men but not Cuban or Puerto Rican women were more likely to report a history of lifetime depression and to be current smokers. Interestingly, education was inversely related to smoking in men but not women. Overall, these results stress the need to consider how vulnerability to mental health and substance use problems can vary for Latino/as who live at the intersection of different social categories, such as ethnicity, gender, class, etc. (Cole 2009).

\section{Limitations and Conclusion}

As with any research, there are limitations to this study. The cross-sectional methodology prevents us from temporally or causally linking lived experiences to the development of major depression and cigarette smoking. However, for the majority of participants, immigration or acculturation likely preceded the onset of MDD and use of cigarettes. That is, $65 \%$ of our sample was either US born $(n=924)$ or had immigrated to the US before the age of $12(n=365)$, and depression and smoking typically came later (mean age of MDD onset $=25.35$ years; mean age of smoking onset $=15.21$ years). In addition, we used not only lifetime measures but also past-year MDD and current smoking, to get a better sense of depression and smoking in the recent past, subsequent to immigration and/or acculturation. Nevertheless, future studies should collect data at different time points, to better understand how acculturation, smoking, and depression unfold over time for women and men.

Although data came from a diverse and representative sample of 2,554 Latino/as, there were not enough cases to consider whether ethnic subgroup interacts with gender and profile group to affect outcomes. Similarly, we worked with smaller cell sizes after stratifying our analysis by gender. Most gender-by-profile groups contained well over 50 cases (see Table 2), but for Groups 4 (37 men) and 5 (44 women), results should be interpreted with caution. Finally, readers should bear in mind the usual limitations that come with self-reported data.

Notwithstanding these limitations, this study advances our understanding of how gender, ethnicity, and acculturation intersect, jointly influencing Latino/a well-being. With profile analyses, we demonstrated the various ways in which lived experiences occur and co-occur, bringing out the diversity of a Latino/a population too often portrayed as one homogenous group. Moreover, we illustrated the associations of different life experience profiles with 
depression and smoking, which provides insight into possible mechanisms linking acculturation to MDD and smoking. We also uncovered both gender differences and similarities.

The results from the present study can inform the development of more targeted intervention, prevention, assessment, and policy-making strategies, tailored to Latino/a men and women from different ethnic backgrounds. Latino/as are at risk for depression and cigarette smoking, and they belong to the largest and fastest-growing immigrant group in the US It is vital to understand why and for whom acculturation relates to increased depression and substance use, and it is equally important to understand why women are more affected than men. This study makes important strides in these directions.

Acknowledgments This work was supported by the University of Michigan Substance Abuse Research Center (UMSARC)/NIDA Predoctoral Training Fellowship (Grant \# T32DA007267). We thank Drs. Albert Cain, Jorge Delva, and Heather Flynn for their advice and mentorship in the execution of this research project.

\section{References}

Agnew, R. (2001). Building on the foundation of general strain theory: Specifying the types of strain most likely to lead to crime and delinquency. The Journal of Research in Crime and Delinquency, 38(4), 319. doi:10.1177/0022427801038004001.

Aguilar-Gaxiola, S., Kramer, E. J., Resendez, C., \& Magaña, C. G. (2008). The context of depression in latinos in the united states. In T. P. Gullotta (Ed.), Depression in U.S. Latinos: Assessment, treatment, and prevention. (pp. 3-28). New York, NY: Springer Science + Business Media.

Alegria, M., Canino, G., Shrout, P. E., Woo, M., Duan, N., Vila, D., et al. (2008). Prevalence of mental illness in immigrant and nonimmigrant U.S. Latino groups. The American Journal of Psychiatry, 165(3), 359-369. doi:10.1176/appi.ajp.2007.07040704.

Alegria, M., Canino, G., Stinson, F. S., \& Grant, B. F. (2006). Nativity and DSM-IV psychiatric disorders among Puerto Ricans, Cuban Americans, and non-Latino whites in the United States: Results from the national epidemiologic survey on alcohol and related conditions. Journal of Clinical Psychiatry, 67(1), 56-65.

Alegria, M., Vila, D., Woo, M., Canino, G., Takeuchi, D., Vera, M., et al. (2004). Cultural relevance and equivalence in the NLAAS instrument: Integrating etic and emic in the development of cross-cultural measures for a psychiatric epidemiology and services study of latinos. International Journal of Methods in Psychiatric Research, 13(4), 270-288. doi:10.1002/mpr.181.

Andrade, L., Caraveo-Anduaga, J., Berglund, P., Bijl, R. V., De Graaf, R., Vollebergh, W., et al. (2003). The epidemiology of major depressive episodes: Results from the international consortium of psychiatric epidemiology (ICPE) surveys. International Journal of Methods in Psychiatric Research, 12(1), 3-21.

Aneshensel, C. S., \& Frerichs, R. R. (1982). Stress, support, and depression: A longitudinal causal model. Journal of Community Psychology, 10, 363-376.

Baer, J. C., \& Schmitz, M. F. (2007). Ethnic differences in trajectories of family cohesion for mexican american and non-hispanic white adolescents. Journal of Youth and Adolescence, 36(4), 583-592. doi:10.1007/s10964-007-9177-3.
Betancourt, H., \& López, S. R. (1993). The study of culture, ethnicity, and race in American psychology. American Psychologist, 48, 629-637. doi:10.1037/0003-066X.48.6.629.

Bethel, J. W., \& Schenker, M. B. (2005). Acculturation and smoking patterns among hispanics: A review. American Journal of Preventive Medicine, 29(2), 143-148.

Breslau, N., Peterson, E. L., Schultz, L. R., Chilcoat, H. D., \& Andreski, P. (1998). Major depression and stages of smoking: A longitudinal investigation. Archives of General Psychiatry, 55(2), 161-166. doi: 10.1001/pubs.ArchGenPsychiatry-ISSN-0003-990x-55-2-yoa6330.

Cabassa, L. J. (2003). Measuring acculturation: Where we are and where we need to go. Hispanic Journal of Behavioral Sciences, 25(2), 127-146. doi:10.1177/0739986303253626.

Center for Disease Control and Prevention (CDC). (2009). Smokingattributable mortality, years of potential life lost, and productivity losses-united states, 2000-2004.

Cole, E. R. (2009). Intersectionality and research in psychology. American Psychologist, 64(3), 170-180. doi:10.1037/a0014564.

Cook, B., Alegría, M., Lin, J. Y., \& Guo, J. (2009). Pathways and correlates connecting latinos' mental health with exposure to the united states. American Journal of Public Health, 99(12), 2247-2254. doi:10.2105/AJPH.2008.137091.

Coonrod, D. V., Balcazar, H., Brady, J., Garcia, S., \& Van Tine, M. (1999). Smoking, acculturation and family cohesion in mexicanAmerican women. Ethnicity and Disease, 9(3), 434-440.

Felix-Ortiz, M., Newcomb, M. D., \& Meyers, H. (1994). A multidimensional measure of cultural identity for Latino and Latina adolescents. Hispanic Journal of Behavioral Sciences, 16(2), 99-115. doi:10.1177/07399863940162001.

Gil, R. M., \& Vazquez, C. I. (1996). The maria paradox. how latinas can merge old world traditions with new world esteem. New York: Putnam.

Grant, B. F., Stinson, F. S., Hasin, D. S., Dawson, D. A., Chou, S. P., \& Anderson, K. (2004). Immigration and lifetime prevalence of DSM-IV psychiatric disorders among mexican americans and non-hispanic whites in the united states: Results from the national epidemiologic survey on alcohol and related conditions. Archives of General Psychiatry, 61(12), 1226-1233. doi: 10.1001/archpsyc.61.12.1226.

Guilamo-Ramos, V., Jaccard, J., Johansson, M., \& Tunisi, R. (2004). Binge drinking among Latino youth: Role of acculturationrelated variables. Psychology of Addictive Behaviors, 18(2), 135. doi:10.1037/0893-164X.18.2.135.

Hartigan, J. A. (Ed.). (1975). Clustering algorithms. Canada: Wiley Series in Probability and Mathematical Statistics.

Heeringa, S. G., Wagner, J., Torres, M., Duan, N., Adams, T., \& Berglund, P. (2004). Sample designs and sampling methods for the collaborative psychiatric epidemiology studies (CPES). International Journal of Methods in Psychiatric Research, 13(4), 221-240. doi:10.1002/mpr.179.

Hovey, J. D., \& Magaña, C. (2000). Acculturative stress, anxiety, and depression among Mexican farmworkers in the Midwest United States. Journal of Immigrant Health, 2(3), 119-131. doi: 10.1023/A:1009556802759.

Kam, J. A., Cleveland, M. J., \& Hecht, M. L. (2010). Applying general strain theory to examine perceived discrimination's indirect relation to mexican-heritage youth's alcohol, cigarette, and marijuana use. Prevention Science: The Official Journal of the Society for Prevention Research, 11(4), 397-410. doi: 10.1007/s11121-010-0180-7.

Kendler, K. S., Neale, M. C., MacLean, C. J., Heath, A. C., Eaves, L. J., \& Kessler, R. C. (1993). Smoking and major depression. A causal analysis. Archives of General Psychiatry, 50(1), 36-43.

Kessler, R. C., \& Ustun, T. B. (2004). The world mental health (WMH) survey initiative version of the world health organization (WHO) composite international diagnostic interview (CIDI). 
International Journal of Methods in Psychiatric Research, 13(2), 93-121. doi:10.1002/mpr.168.

Lakes, K., López, S. R., \& Garro, L. (2006). Cultural competence and psychotherapy: Applying anthropologically informed conceptions of culture. Psychotherapy: Theory, Research, Practice, Training, 43(4), 380-396. doi:10.1037/0033-3204.43.4.380.

López, S. R., \& Guarnaccia, P. J. J. (2000). Cultural psychopathology: Uncovering the social world of mental illness. Annual Review of Psychology, 51, 571-598. doi:10.1146/annurev.psych.51.1.571.

Magnusson, D. (1998). The logic and implications of a personcentered approach. In R. B. Cairns (Ed.), Methods and models for studying the individual (pp. 33-64). Thousand Oaks, CA: Sage.

Miranda, A. O., Estrada, D., \& Firpo-Jimenez, M. (2000). Differences in family cohesion, adaptability, and environment among Latino families in dissimilar stages of acculturation. The Family Journal, 8(4), 341-350. doi:10.1177/1066480700084003.

Olson, D. H. (1986). Circumplex model VII: Validation studies and FACES III. Family Process, 25, 337-351. doi:10.1111/j.15455300.1986.00337.x.

Ong, A. D., Fuller-Rowell, T., \& Burrow, A. L. (2009). Racial discrimination and the stress process. Journal of Personality and Social Psychology, 96(6), 1259-1271. doi:10.1037/a0015335.

Pérez, D. J., Fortuna, L., \& Alegría, M. (2008). Prevalence and correlates of everyday discrimination among U.S. latinos. Journal of Community Psychology, 36(4), 421-433. doi: 10.1002/jcop.20221.

Pérez-Stable, E. J., Ramirez, A., Villareal, R., Talavera, G. A., Trapido, E., Suarez, L., et al. (2001). Cigarette smoking behavior among US Latino men and women from different countries of origin. American Journal of Public Health, 91(9), 1424-1430.

Pratt, L., \& Brody, D. (2010). Depression and smoking in the U.S. household population aged 20 and over, 2005-2008. NCHS Data Brief, 34, 1-8.
Quattrocki, E., Baird, A., \& Yurgelun-Todd, D. (2000). Biological aspects of the link between smoking and depression. Harvard Review of Psychiatry, 8(3), 99-110.

Rivera, F. I. (2007). Contextualizing the experience of young Latino adults: Acculturation, social support and depression. Journal of Immigrant and Minority Health, 9(3), 237-244. doi: 10.1007/s10903-006-9034-6.

Rivera, F. I., Guarnaccia, P. J., Mulvaney-Day, N., Lin, J. Y., Torres, M., \& Alegría, M. (2008). Family cohesion and its relationship to psychological distress among Latino groups. Hispanic Journal of Behavioral Sciences, 30(3), 357-378. doi:10.1177/07399863 08318713 .

Sarmiento, I. A., \& Cardemil, E. V. (2009). Family functioning and depression in low-income Latino couples. Journal of Marital and Family Therapy, 35(4), 432-445. doi:10.1111/j.1752-0606. 200901139.x.

Schwartz, S. J., Unger, J. B., Zamboanga, B. L., \& Szapocznik, J. (2010). Rethinking the concept of acculturation: Implications for theory and research. American Psychologist, 65(4), 237-251. doi:10.1037/a0019330.

U.S. Census Bureau. (2010). U.S. Census Bureau News: Facts for features: Hispanic heritage month 2010. Retrieved April 01, 2011, from http://www.census.gov/newsroom/releases/archives/ facts_for_features_special_editions/.

Vega, W. A., \& Sribney, W. M. (2008). Latino population demographics, risk factors, and depression: A case study of the Mexican American prevalence and services survey. In T. P. Gullotta (Ed.), Depression in Latinos: Assessment, treatment, and prevention (pp. 29-52). New York, NY, USA: Springer Science + Business Media.

Wiehe, S. E., Aalsma, M. C., Liu, G. C., \& Fortenberry, J. D. (2010). Gender differences in the association between perceived discrimination and adolescent smoking. American Journal of Public Health, 100(3), 510-516. doi:10.2105/AJPH.2009.169771. 